\title{
Question Of Determining Diagnostics Time Intervals Of Electric Transport Systems Using Time Between Failures
}

\author{
B.V. Malozyomov \\ Novosibirsk State Technical University \\ Novosibirsk, Russian Federation \\ mbv5@mail.ru \\ M.V. Rozhkova \\ Novosibirsk State Technical University \\ Novosibirsk, Russian Federation \\ marina.rozhkova.63@mail.ru
}

\author{
M.E. Vilberger \\ Novosibirsk State Technical University \\ Novosibirsk, Russian Federation \\ vilberger@corp.nstu.ru \\ A.A. Shtang \\ Novosibirsk State Technical University \\ Novosibirsk, Russian Federation \\ shtang@corp.nstu.ru
}

\begin{abstract}
The determination of the current technical resource of the electric transport system is an important characteristic that allows one to define not only the current reliability of the system, but also to predict it for the future. If the maintenance of electrical equipment is carried out to restore the reliability, failure flow is not stopped, then the intensity of equipment failure will be artificially overstated. The timing of service impacts will also be overstated unsuccessfully.
\end{abstract}

Keywords- diagnostics, reliability, electromechanical systems, electric transport.

\section{INTRODUCTION}

Determination of the current technical resource of electric transport systems is an important characteristic that allows one to determine not only the current reliability of systems, but also to predict it for the future. If the maintenance of electrical equipment $(\mathrm{EE})$ is carried out with the restoration of reliability up $P(t) \approx 1$ to period I (Fig. 1), then the flow of run-in failures is not stopped, the intensity of equipment failure will be artificially overstated. Obviously, the timing of service impacts will also be erroneously overstated.

If during the preventive maintenance, the reliability was restored up $P(t) \approx 1$, the frequency of prevention was equal to $t_{d}$ and it was carried out for each $N$ units of EE of one group evenly with interval $t_{\partial} / N$, then the reliability curves of each object will have form (2), where the qualitative dependences for a group of homogeneous objects are given where $N=5$. Naturally, the assumption of recovery in the prevention up to $P(t) \approx 1$ is approximate.

\section{MATHEMATICAL DESCRIPTION OF OBJECT}

In the discussion group, reliability $k$ of each object ( $k=0,1,2, \ldots, N-1$ ), taking into account that the worn out trolleybus fleet, dominated by the exponential law of reliability, is determined by the expression:

$$
P_{k}(t)=e^{-\frac{t+k \cdot\left(\frac{t_{d}}{N}\right)}{T}},
$$

where $T$ - time of failure of the $k$ element. In this case, the average reliability function of the entire group consisting of $N$ objects in the interval of $t=0 \ldots \frac{t_{d}}{N}$ is represented by the expression:

$$
\overline{P(t)}=\frac{P_{1}(t)+P_{2}(t)+\ldots+P_{k}(t)+\ldots+P_{N}(t)}{N} .
$$

Recording (2) as the sum of the geometric progression divided by $N$, there will be the following:

$$
\overline{P(t)}=\frac{e^{\frac{t_{d}-N\left(t+t_{d}\right)}{N \cdot T}} \cdot\left(1-e^{-\frac{t_{d}}{T}}\right)}{N \cdot\left(1-e^{-\frac{t_{d}}{N \cdot T}}\right)}
$$

Thus, the value of the average reliability of the entire group of objects for the year can be analytically determined from (3)

$$
\overline{P_{a v}}=\frac{\overline{P(t)_{\max }}+\overline{P(t)}}{2}
$$

For the values of time $t=0$ and $t=\frac{t_{d}}{N}$, determining the maximum and minimum level of reliability of each of the 
objects $N$ in the group, there will be the following:

$$
\overline{P(t)_{\max }}=\frac{e^{\frac{t_{d} \cdot(1-N)}{N \cdot T}} \cdot\left(1-e^{-\frac{t_{d}}{T}}\right)}{N \cdot\left(1-e^{\left.-\frac{t_{d}}{N \cdot T}\right)}\right.}{\overline{P(t)_{\min }}}^{=} \frac{e^{-\frac{t_{d}}{T}} \cdot\left(1-e^{-\frac{t_{d}}{T}}\right)}{N \cdot\left(1-e^{-\frac{t_{d}}{N \cdot T}}\right)}
$$

From equations (4) and (5) with failure rate $\lambda=\frac{1}{T}$, the expression can be obtained for $\overline{P_{a v}}$.

As shown by experiments, annual fluctuations of the failure parameter take place. In this regard, there are confidence limits, in which the actual magnitude of the failure rate of the element under consideration can exist. Confidence limits are determined for the likelihood of their compliance failures are random occurrences and there is a possibility of out of range of $\lambda$. In this regard $\alpha$ is used as the confidence factor. Practically assuming that the probability of failure of the failure rate beyond the confidence interval is $90 \%$ ( $\alpha=0.9$ ), the definition of lower $\lambda_{n}$ and upper $\lambda_{b}$ limits of the confidence interval is:

$$
\lambda_{n, b}=\frac{\lambda}{r_{1,2}}
$$

where $r_{1,2}$ - are specially tabulated functions, depending on the number of failures $m$ and coefficient $\alpha$ [124]. The upper limit of the failure rate is of practical interest, the failure rate is maximal.

The results of calculating failure rate $\lambda$ and the mean time between failures $T$ for the electrical complex of a trolleybus in Novosibirsk are shown in Table I.

Using the data of Table $\mathrm{I}$, it is possible to distinguish a group of electrical equipment where significant increases in the failure rate are possible: motor-compressor, line contactor 5 and 4, circuit breaker, starting and braking resistors, pedals, alarms and batteries.

With high quality of service impacts, an increase in failure rates $\lambda$ for the rest of the electrical equipment with a $90 \%$ probability will not exceed $10-12 \%$ (Table I).

Using equations (5) in (4), the equation will be obtained allowing defining its individual failure rates $\lambda$ in given operating conditions having an average object reliability during a year.

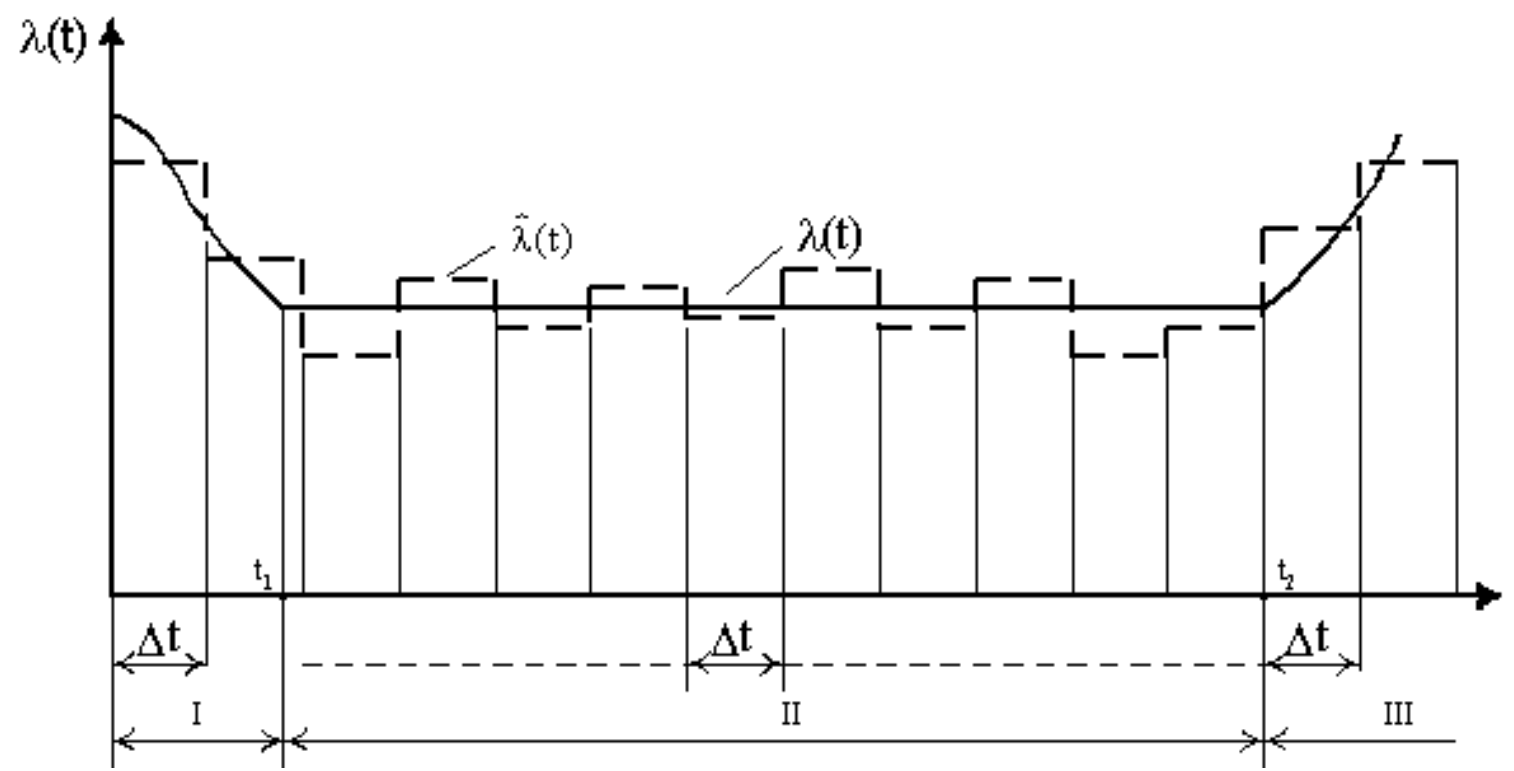

Fig. 1. Change in the failure rate of electrical systems of the trolleybus over a long period of operation: I - the period of run-in, II - long-term operation; III - the period before the overhaul. 


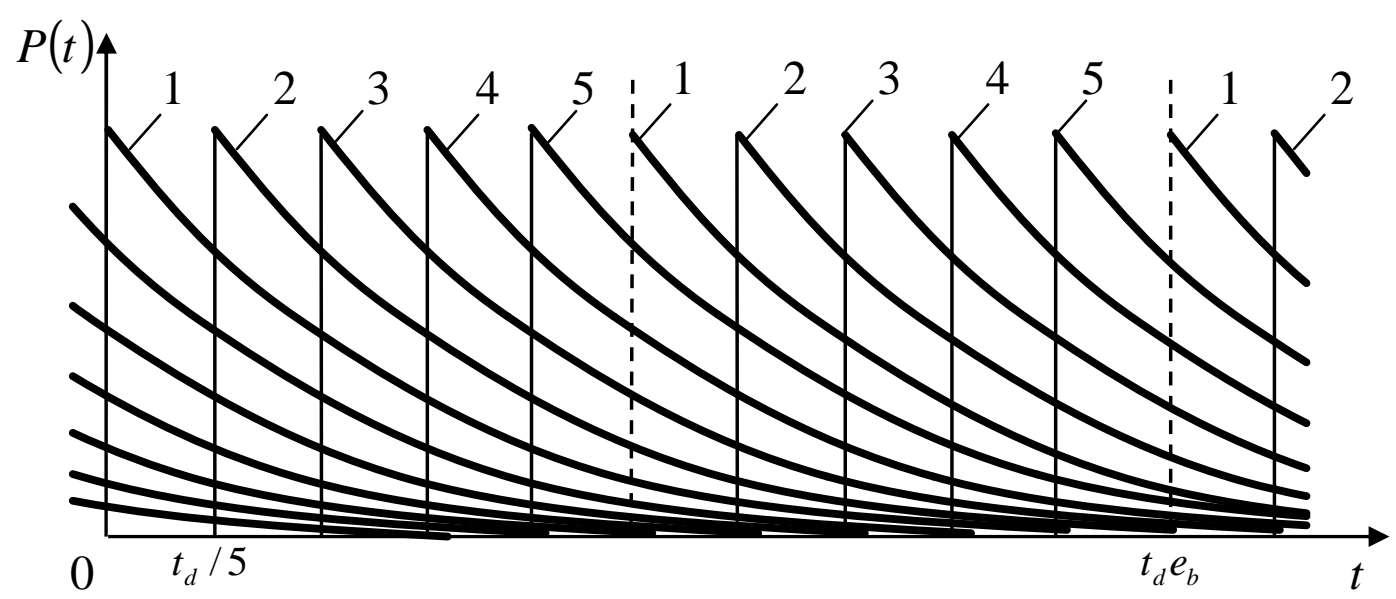

Fig. 2. Change of reliability of objects in the system with periodic diagnostics and recovery up to $P(t)=1$

\begin{tabular}{|c|c|c|c|c|c|c|}
\hline № & Type of electrical equipment & $\begin{array}{c}\lambda, \\
1 / \text { year }\end{array}$ & $\begin{array}{c}\alpha=0.9 \\
\text { Confidence interval }\end{array}$ & $\begin{array}{c}T, \\
\text { year }\end{array}$ & $t_{\text {doptimal }}$, & $k_{\text {returning }}$ \\
\hline
\end{tabular}

$$
\bar{P}_{s r}=\frac{\left(1-e^{-\frac{t_{d}}{T}}\right)}{2 \cdot N \cdot\left(1-e^{-\frac{t_{d}}{N \cdot T}}\right)} \cdot\left(e^{\frac{t_{d \cdot(1-N)} \cdot T}{N \cdot T}}+e^{-\frac{t_{d}}{T}}\right)
$$

where $T=\frac{1}{\lambda}$. Equation (7) is transcendental with respect to $T$, which is solved only by numerical methods. Let us solve it for one point by the graphic method. Taking into account the smallness of exponent $t_{d} / N \cdot T$ when $N=100$ of mobile units, according to [125]:

$$
e^{-\frac{t_{d}}{N \cdot T}} \approx 1-\frac{t_{d}}{T \cdot N}
$$

Let us obtain a simpler equation:

$$
\bar{P}_{s r}=\frac{\left(1-e^{-\frac{t_{d}}{T}}\right) \cdot\left(e^{\frac{t_{d \cdot(1-N)}}{N \cdot T}}+e^{-\frac{t_{d}}{T}}\right)}{2 \cdot \frac{t_{d}}{T}}
$$

Given $N=100$ with expression $1-N \approx-N$, one gets

$$
\bar{P}_{a v} \cdot \frac{t_{d}}{T}=\left(1-e^{-\frac{t_{d}}{T}}\right) \cdot e^{-\frac{t_{d}}{T}}
$$

Equation (10) is solved graphically, determining the intersection point of the graphs $\left(1-e^{-\frac{t_{d}}{T}}\right) \cdot e^{-\frac{t_{d}}{T}}$ and $\bar{P}_{s r} \cdot \frac{t_{d}}{T}$

The graphical solution of equation (7) is shown in Fig. 3. Thus, for certain $P=0.921$ and $t_{d}=3.5$, let us find $T=20.9$.

Using mathematical-analytical software packages MathCAD Enterprise Edition 11.0, Wolfram research Mathematica 5.0, Waterloo Maple $8.0 t_{d}$, families of characteristics and a three-dimensional surface in coordinates $\left(\bar{P}_{s r}, T, t_{d}\right)$ were obtained.

In Fig. 4 graphs are shown for determining the individual operating time for failure with given reliability of the whole group and the time of restoration of electrical equipment to $P=1$ when $t_{d}=3.5$ day, when $t_{d}=7$ day, when $t_{d}=14$ day, when $t_{d}=28$ day, when $t_{d}=56$ day, when $t_{d}=112$ day, when $t_{d}=224$ day, corresponding to a complete repair cycle. Fig. 5 shows the solution of equation (7) with respect to a 3-dimensional surface in coordinates $\left(\bar{P}_{s r}, T, t_{d}\right)$ when $T=1 \ldots 1000$ days, $\bar{P}_{s r}=0.1 \ldots 0.95$ and $t_{d}=0 \ldots 1600$ days, materials are given in Table II. 


\begin{tabular}{|c|c|c|c|c|c|c|c|}
\hline & & & $\lambda_{n}$ & $\lambda_{v}$ & & & \\
\hline 1. & circuit breaker & 2.015 & 1.914 & 2.156 & 0.46 & 2.31 & 0.225 \\
\hline 2. & traction motor & 3.235 & 3.073 & 3.461 & 0.28 & 0.72 & 0.218 \\
\hline 3. & auxiliary engine & 7.167 & 6.808 & 7.668 & 0.13 & 0.32 & 0.322 \\
\hline "4."' & motor compressor & 6.123 & 5.816 & 6.551 & 0.15 & 19.5 & 0.250 \\
\hline 5. & generator & 3.055 & 2.902 & 3.268 & 0.30 & 0.76 & 0.186 \\
\hline 6. & regulating relay & 3.414 & 3.243 & 3.652 & 0.27 & 0.68 & 0.272 \\
\hline 7. & voltage-response relay & 3.235 & 3.073 & 3.461 & 0.28 & 0.72 & 0.274 \\
\hline 8. & current relay & 3.305 & 3.139 & 3.536 & 0.27 & 0.71 & 0.003 \\
\hline 9 & collector bow & 9.58 & 9.101 & 10.25 & 0.09 & 0.16 & 0.163 \\
\hline 10. & control line & 3.235 & 3.073 & 3.461 & 0.28 & 0.72 & 0.139 \\
\hline 11. & electric contactor & 17.85 & 16.95 & 19.09 & 0.05 & 0.13 & 0.197 \\
\hline 12. & shunt field rheostat & 2.575 & 2.446 & 2.755 & 0.36 & 0.91 & 0.419 \\
\hline 13. & line contactor 4 and 5 & 1.667 & 1.583 & 1.783 & 0.56 & 3.08 & 0.091 \\
\hline 14. & ampere drain & 4.735 & 4.498 & 5.066 & 0.19 & 0.49 & 0.393 \\
\hline 15. & reverse controller & 4.378 & 4.159 & 4.684 & 0.21 & 0.53 & 0.233 \\
\hline 16. & cell & 4.735 & 4.498 & 5.066 & 0.19 & 0.85 & 0.296 \\
\hline 17. & foot bar & 5.405 & 5.134 & 5.783 & 0.17 & 1.65 & 0.192 \\
\hline 18. & signaling system & 0.915 & 0.869 & 0.979 & 1.02 & 1.75 & 0.136 \\
\hline 19. & servo-motor & 6.215 & 5.904 & 6.650 & 0.15 & 0.37 & 0.135 \\
\hline 20. & furnace and lightning & 3.355 & 3.187 & 3.589 & 0.27 & 0.69 & 0.187 \\
\hline
\end{tabular}

TABLE I. THE FAILURE RATE AND THE MEAN TIME BETWEEN FAILURES OF THE ELECTRICAL COMPLEX OF A TROLLEYBUS

TABLE II. VALUES OF INDIVIDUAL TIME BETWEEN FAILURES WITH GIVEN RELIABILITY AND RECOVERY TIME OF ELECTRICAL EQUIPMENT

\begin{tabular}{|c|c|c|c|c|c|c|c|}
\hline \multirow{2}{*}{$\bar{P}_{s r}$} & \multicolumn{7}{|c|}{$T$, days } \\
\hline & $t_{d}=3.5$ & $t_{d}=7$ & $t_{d}=14$ & $t_{d}=28$ & $t_{d}=56$ & $t_{d}=112$ & $t_{d}=224$ \\
\hline 0.01 & 1.031 & 2.062 & 4.131 & 8.271 & 16.542 & 33.084 & 66.169 \\
\hline 0.1 & 2.164 & 4.328 & 8.658 & 17.316 & 34.633 & 69.265 & 138.53 \\
\hline 0.2 & 3.141 & 6.2822 & 12.561 & 25.122 & 50.244 & 100.489 & 200.98 \\
\hline 0.3 & 4.231 & 8.462 & 16.927 & 33.854 & 67.709 & 135.42 & 270.84 \\
\hline 0.4 & 5.592 & 11.184 & 22.368 & 44.737 & 89.474 & 178.95 & 357.89 \\
\hline 0.5 & 7.424 & 14.849 & 29.699 & 59.397 & 118.79 & 237.59 & 475.18 \\
\hline 0.6 & 10.112 & 20.224 & 40.441 & 80.882 & 161.76 & 323.53 & 647.05 \\
\hline 0.7 & 14.522 & 29.051 & 58.091 & 116.18 & 232.36 & 464.72 & 929.45 \\
\hline 0.8 & 23.272 & 46.541 & 93.089 & 186.18 & 372.36 & 744.71 & 1489.4 \\
\hline 0.9 & 49.392 & 98.797 & 197.594 & 395.19 & 790.38 & 1580.8 & 3161.5 \\
\hline 0.99 & 518.79 & 1037.6 & 2075.1 & 4150.3 & 8300.6 & 16601 & 33202 \\
\hline
\end{tabular}

TABLE III. DEPENDENCE BETWEEN THE FAILURE RATE AND THE DURATION OF RUNNING-IN OF THE SWITCHING ELECTRICAL EQUIPMENT OF THE TROLLEYBUS

\begin{tabular}{|c|c|c|}
\hline № & Name of equipment & $T_{\text {run }}$, days \\
\hline 1. & circuit breaker & 1.51 \\
\hline 2. & regulating relay & 1.24 \\
\hline 3. & voltage-response relay & 1.15 \\
\hline 4. & current relay & 1.08 \\
\hline 5. & drive circuit & 1.49 \\
\hline 6. & line contactor LC4, LC5 & 2.20 \\
\hline 7. & Rheostat reverse controller & 1.83 \\
\hline 8. & master controller & 2.15 \\
\hline 9. & field weakening contactor & 1.71 \\
\hline
\end{tabular}




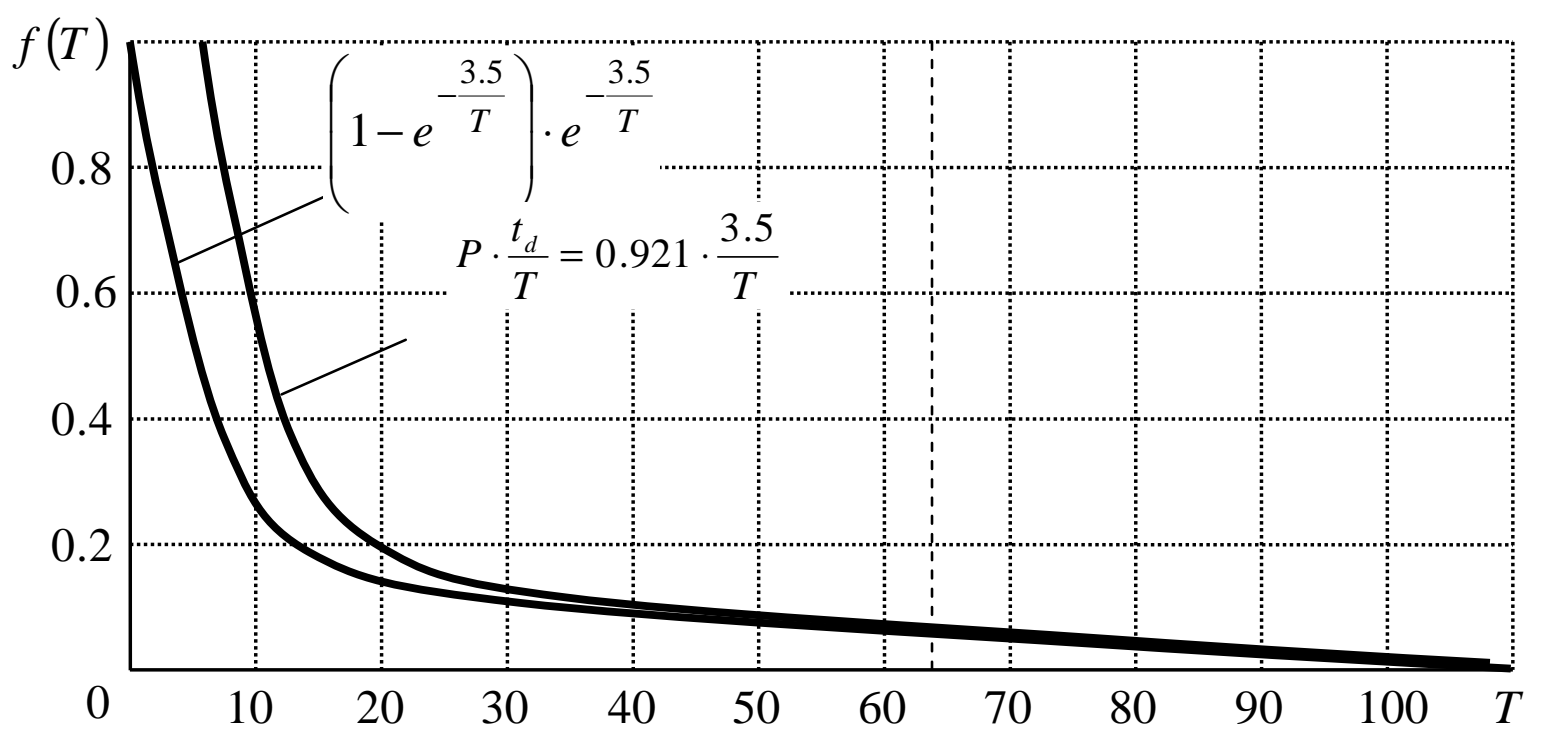

Fig. 3. Graphic determination of the operating time to failure $T$ with a known average for a year of reliability $P$ and diagnosis period $t_{d}$

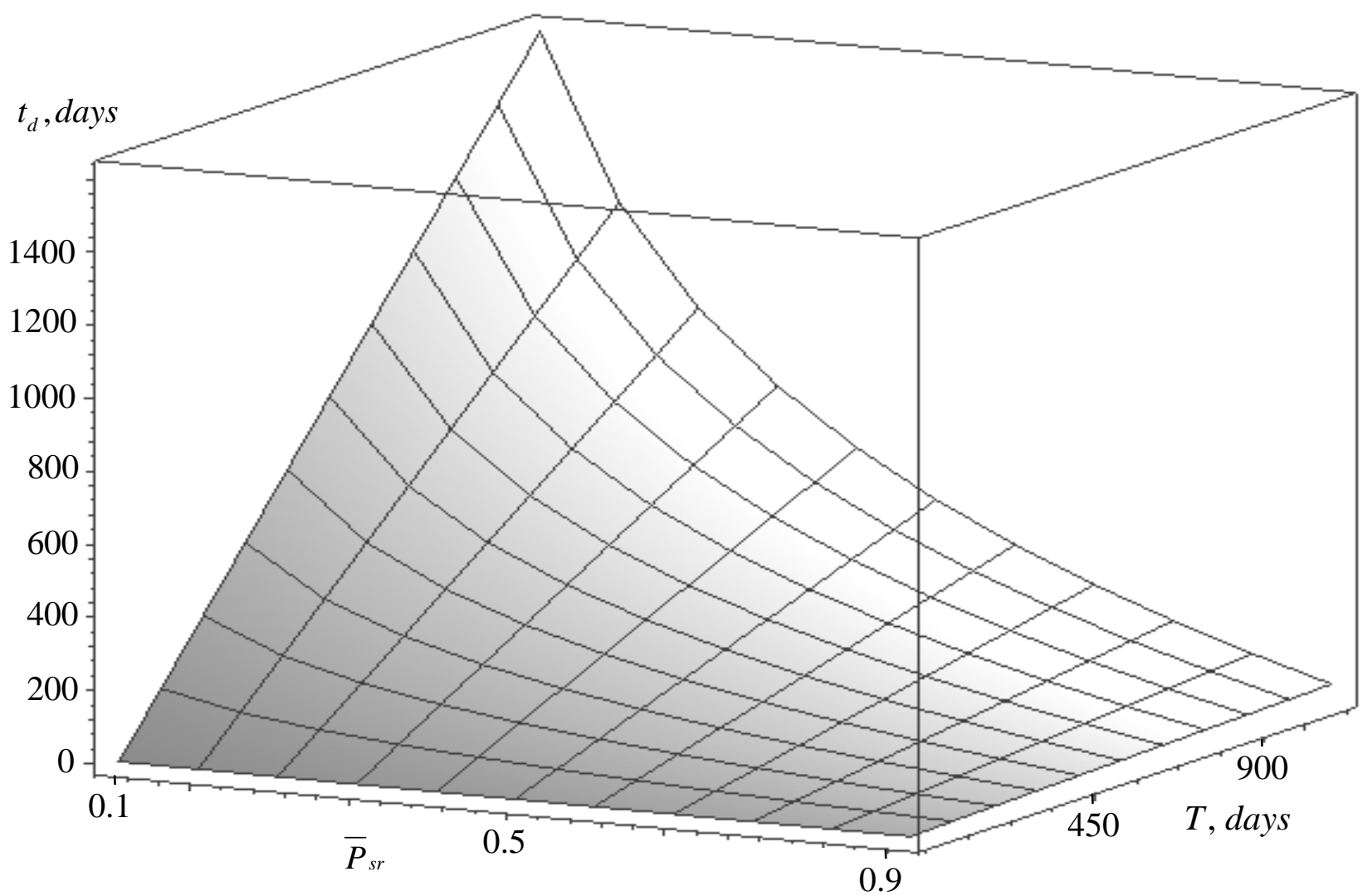

Fig. 4. 3D surface $\left(\bar{P}_{s r}, T, t_{d}\right)$ to define $T$ with given $t_{d}$ and $\bar{P}_{s r}$ 


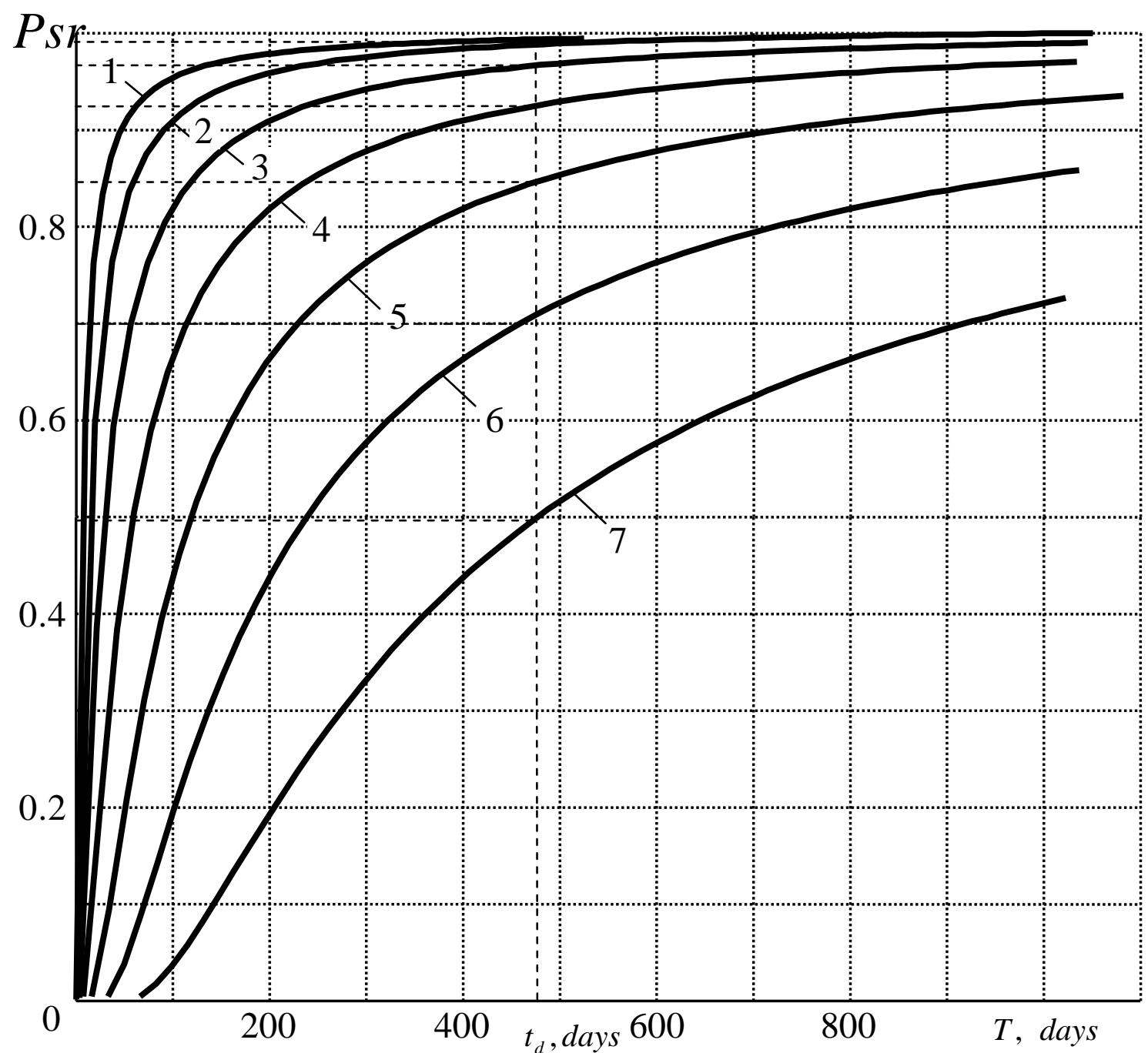

Fig. 5. Dependence between reliability during year $\overline{P_{s r}}$ and operating time for failure $T$ when: $1-t_{d}=3.5$ day, $2-t_{d}=7$ days, $3-t_{d}=14$ days, $4-t_{d}=28$ days, $5-t_{d}=56$ days, $6-t_{d}=112$ days, $7-t_{d}=224$ days 


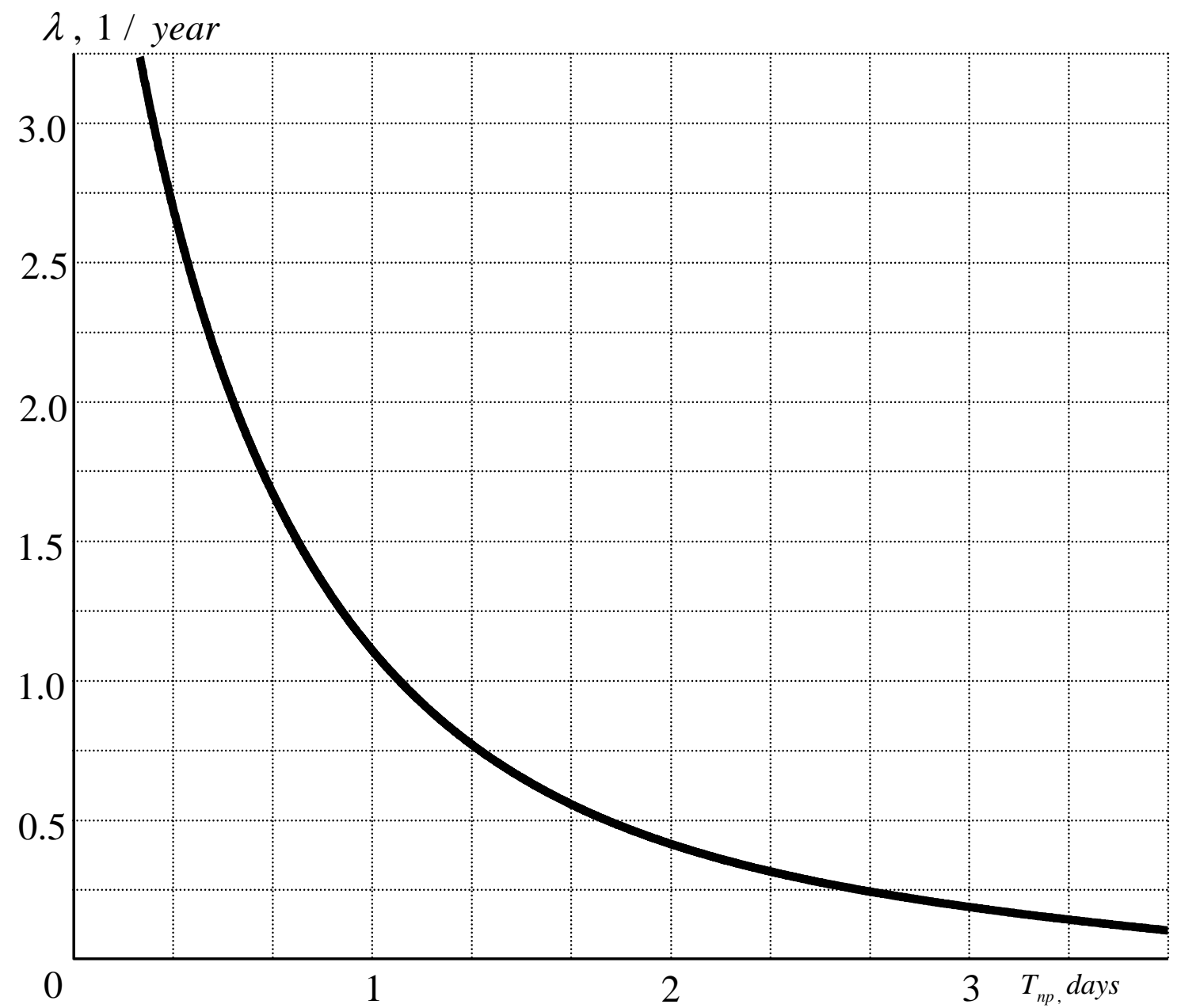

Fig. 6. Dependence between failure rate $\lambda$ and duration of running-in $T_{r u n}$ of the switching electrical equipment of the trolleybus

Obviously, if the time between failures is determined by the most dangerous border (Table III) external conditions and operating factors, the duration of the running-in of the trolleybus switching equipment was determined.

$$
T_{v}=\frac{1}{\lambda_{v}}
$$

is a year or a significant part of it, the use of the graphs in Fig. 4 and Fig. 5 gives almost the same value as the expression (11). With smaller value $T_{v}$ Fig. 4 gives a slightly different, more accurate value of the operating time. From Fig. 4 it is seen that for a given time between failures for a traction motor $T_{1}=476$ at different diagnostic time values $t_{d}$. For example for a traction motor, one will have a different level of its reliability. So, for $t_{d}=28$ days, let us have $\bar{P}_{s r}=0.921$, but for $t_{d}=7$ a day, let us have $\bar{P}_{s r}=0.96$. Thus, it is obvious that when the diagnostic time decreases, the reliability of the electrical equipment of the trolleybus increases. For the electrical engineering complexes of the trolleybus in Fig. 6, there is a graph of the relationship between failure rates $\lambda$ and the period of running-in of the switching electrical equipment of the trolleybus [135] During the run-in period, the objects have a significant failure rate. Taking the intensity of failures for the measure of the intensity of operation and taking into account the quality of service impacts, environmental conditions and real-world effects the period of running-in of electrical equipment of trolleybus were determined.

\section{RESEARCH RESULTS}

Thus, the data obtained with the average time of 
prevention are overestimated because of the large proportion of working hours in the entire preventive period. Obviously, this conclusion should serve as a prerequisite for a certain refinement of maintenance time for each of the types of $\mathrm{EE}$ trolleybus. At the same time, due to the fact that the estimates of the working hours are based on the failure ratio, these values can be slightly increased and, for example, the running time for the repaired contactor of field weakening will slightly exceed the time of 1.7 day and night.

The foregoing practically means that it is impossible to carry out adjusting operations, for example, in an efficient control circuit more than once every 6 day and night, since the danger of failure of such regulation is undoubtedly increased because the entire control circuit again starts operating its elements. Moreover, in the presence of a control circuit failure, it is important to find a really faulty element, without violating substantially the operating conditions of other elements that are already in the ready-made state, that is, in the mode according to Fig. 1, with a low failure rate. At the same time, the running-in time should be a small fraction of the total time of the interdiagnostic period (Fig. 2).

So, if initial running-in intensity $\lambda$ is 3 times higher than the intensity during normal operation of the $\mathrm{EE}$, then for an interdiagnostic period, $t_{d}$ equals three times the running-in time $T_{r u n}$, the average failure rate will be equal to $1.33 \cdot \lambda$. This means that the probability per unit of failure time in the EE group due to the large share of still unprocessed elements will be increased by $33 \%$ relative to the situation when all the elements in the given group have already been worked on.

\section{CONCLUSION}

Thus, according to the calculations, for some elements of the EE trolley bus, it is necessary to increase the existing interprofessional period.

The methods and criteria set forth in the example of aircraft electrical equipment maintenance allowed clarifying this conclusion.

\section{References}

[1] O.I. Sablin, "Improving the efficiency of energy recovery in the electric transport system with limited traction power supply", Technological audit and production reserves, vol. 5/1, pp. 21-26, 2014.

[2] V. G. Sysenko VG, D.A. Bosvit, E.N. Kosarev, "Improvement of the methodology for calculating the distributed traction power-supply system with an amplifying point", Power electronics and power engineering, vol. 9, pp. 8-18, 2014.

[3] V.V. Biryukov, A.V. Kulekina, "The calculation features of the electrical energy storage devices parameters in transport", IFOST 2016, pp. 41-43, June 2016 [The 11 International forum on strategic technology, p. 596, 2016].

[4] V.I. Sopov, N.I. Schurov, Y.A. Prokushev and A.A. Shtang, "Increasing the efficiency of the use of electrical energy in the sub-subsystem of electric transport", Improvement of technical means of electric transport, vol. 3 (29), pp. 115-123, Jule 2002 [Digest of scientific works of the NSTU, p. 189, 2002].

[5] E.N. Bykov, B.V.Panin, V.N.Pupynin, Traction networks of subways, Moskow: Transport, 1987.

[6] M.V. Tarnizhevsky, D.K. Tomlyakovich, Design of power supply devices for trams and trolleybuses, Moskow: Transport, 1986.

[7] K.G. Marquardt, Electrosupply of Electrified Railways, Moskow: Transport, 1982.

[8] M.A. Sleptsov, Fundamentals of Electric Transport, Moskow: Academy, 2006.

[9] L.A. Bessonov, Theoretical bases of electrical engineering, Moscow: Higher School, 1973.

[10] V.I.Sopov, V.V. Biryukov, Y.A. Prokushev, Y.A. Rylov, "Analysis of power supply systems for rolling stock with various schemes of traction networks", Abstract journal of VINITI RAS: Transport, Science, Engineering, Management, vol. 2 (2008), pp. 49-53, 2008. 
